hep-ex/0408022

BABAR-CONF-04/21

SLAC-PUB-10618

August 2004

\title{
Measurement of the Branching Fraction of $e^{+} e^{-} \rightarrow B^{0} \bar{B}^{0}$ at the $\Upsilon(4 S)$ Resonance
}

\author{
The BABAR Collaboration
}

August 10, 2004

We report a measurement of the branching fraction $e^{+} e^{-} \rightarrow B^{0} \bar{B}^{0}$ with a data sample of $81.7 \mathrm{fb}^{-1}$ collected at the $\Upsilon(4 S)$ resonance with the BABAR detector at the PEP-II asymmetricenergy $e^{+} e^{-}$storage ring. Using partial reconstruction of the decay $\bar{B}^{0} \rightarrow D^{*+} \ell^{-} \bar{\nu}_{\ell}$ we obtain a preliminary result of $f_{00}=0.486 \pm 0.010$ (stat.) \pm 0.009 (sys.). Our result does not depend on branching fractions of the $\bar{B}^{0}$ and the $D^{*+}$ decay chains, on the simulated reconstruction efficiency, on the ratio of the charged and neutral $B$ meson lifetimes, nor on assumption of isospin symmetry. This measurement is important for normalizing many $B$ decay branching fractions, and contributes to our understanding of isospin violation in the $\Upsilon(4 S)$ system.

Submitted to the $32^{\text {nd }}$ International Conference on High-Energy Physics, ICHEP 04, 16 August-22 August 2004, Beijing, China

Stanford Linear Accelerator Center, Stanford University, Stanford, CA 94309

Work supported in part by Department of Energy contract DE-AC03-76SF00515. 
The BABAR Collaboration,

B. Aubert, R. Barate, D. Boutigny, F. Couderc, J.-M. Gaillard, A. Hicheur, Y. Karyotakis, J. P. Lees, V. Tisserand, A. Zghiche

Laboratoire de Physique des Particules, F-74941 Annecy-le-Vieux, France

A. Palano, A. Pompili

Università di Bari, Dipartimento di Fisica and INFN, I-70126 Bari, Italy

J. C. Chen, N. D. Qi, G. Rong, P. Wang, Y. S. Zhu

Institute of High Energy Physics, Beijing 100039, China

G. Eigen, I. Ofte, B. Stugu

University of Bergen, Inst. of Physics, N-5007 Bergen, Norway

G. S. Abrams, A. W. Borgland, A. B. Breon, D. N. Brown, J. Button-Shafer, R. N. Cahn, E. Charles, C. T. Day, M. S. Gill, A. V. Gritsan, Y. Groysman, R. G. Jacobsen, R. W. Kadel, J. Kadyk, L. T. Kerth,

Yu. G. Kolomensky, G. Kukartsev, G. Lynch, L. M. Mir, P. J. Oddone, T. J. Orimoto, M. Pripstein, N. A. Roe, M. T. Ronan, V. G. Shelkov, W. A. Wenzel

Lawrence Berkeley National Laboratory and University of California, Berkeley, CA 94720, USA

M. Barrett, K. E. Ford, T. J. Harrison, A. J. Hart, C. M. Hawkes, S. E. Morgan, A. T. Watson

University of Birmingham, Birmingham, B15 2TT, United Kingdom

M. Fritsch, K. Goetzen, T. Held, H. Koch, B. Lewandowski, M. Pelizaeus, M. Steinke

Ruhr Universität Bochum, Institut für Experimentalphysik 1, D-44780 Bochum, Germany

J. T. Boyd, N. Chevalier, W. N. Cottingham, M. P. Kelly, T. E. Latham, F. F. Wilson

University of Bristol, Bristol BS8 1TL, United Kingdom

T. Cuhadar-Donszelmann, C. Hearty, N. S. Knecht, T. S. Mattison, J. A. McKenna, D. Thiessen

University of British Columbia, Vancouver, BC, Canada V6T $1 Z 1$

A. Khan, P. Kyberd, L. Teodorescu

Brunel University, Uxbridge, Middlesex UB8 3PH, United Kingdom

A. E. Blinov, V. E. Blinov, V. P. Druzhinin, V. B. Golubev, V. N. Ivanchenko, E. A. Kravchenko,

A. P. Onuchin, S. I. Serednyakov, Yu. I. Skovpen, E. P. Solodov, A. N. Yushkov

Budker Institute of Nuclear Physics, Novosibirsk 630090, Russia

D. Best, M. Bruinsma, M. Chao, I. Eschrich, D. Kirkby, A. J. Lankford, M. Mandelkern, R. K. Mommsen, W. Roethel, D. P. Stoker

University of California at Irvine, Irvine, CA 92697, USA

C. Buchanan, B. L. Hartfiel

University of California at Los Angeles, Los Angeles, CA 90024, USA

S. D. Foulkes, J. W. Gary, B. C. Shen, K. Wang

University of California at Riverside, Riverside, CA 92521, USA 
D. del Re, H. K. Hadavand, E. J. Hill, D. B. MacFarlane, H. P. Paar, Sh. Rahatlou, V. Sharma University of California at San Diego, La Jolla, CA 92093, USA

J. W. Berryhill, C. Campagnari, B. Dahmes, O. Long, A. Lu, M. A. Mazur, J. D. Richman, W. Verkerke University of California at Santa Barbara, Santa Barbara, CA 93106, USA

T. W. Beck, A. M. Eisner, C. A. Heusch, J. Kroseberg, W. S. Lockman, G. Nesom, T. Schalk, B. A. Schumm, A. Seiden, P. Spradlin, D. C. Williams, M. G. Wilson

University of California at Santa Cruz, Institute for Particle Physics, Santa Cruz, CA 95064, USA

J. Albert, E. Chen, G. P. Dubois-Felsmann, A. Dvoretskii, D. G. Hitlin, I. Narsky, T. Piatenko, F. C. Porter, A. Ryd, A. Samuel, S. Yang

California Institute of Technology, Pasadena, CA 91125, USA

S. Jayatilleke, G. Mancinelli, B. T. Meadows, M. D. Sokoloff

University of Cincinnati, Cincinnati, $\mathrm{OH}$ 45221, USA

T. Abe, F. Blanc, P. Bloom, S. Chen, W. T. Ford, U. Nauenberg, A. Olivas, P. Rankin, J. G. Smith, J. Zhang, L. Zhang

University of Colorado, Boulder, CO 80309, USA

A. Chen, J. L. Harton, A. Soffer, W. H. Toki, R. J. Wilson, Q. L. Zeng

Colorado State University, Fort Collins, CO 80523, USA

D. Altenburg, T. Brandt, J. Brose, M. Dickopp, E. Feltresi, A. Hauke, H. M. Lacker, R. Müller-Pfefferkorn, R. Nogowski, S. Otto, A. Petzold, J. Schubert, K. R. Schubert, R. Schwierz, B. Spaan, J. E. Sundermann

Technische Universität Dresden, Institut für Kern- und Teilchenphysik, D-01062 Dresden, Germany

D. Bernard, G. R. Bonneaud, F. Brochard, P. Grenier, S. Schrenk, Ch. Thiebaux, G. Vasileiadis, M. Verderi Ecole Polytechnique, LLR, F-91128 Palaiseau, France

D. J. Bard, P. J. Clark, D. Lavin, F. Muheim, S. Playfer, Y. Xie

University of Edinburgh, Edinburgh EH9 3JZ, United Kingdom

M. Andreotti, V. Azzolini, D. Bettoni, C. Bozzi, R. Calabrese, G. Cibinetto, E. Luppi, M. Negrini, L. Piemontese, A. Sarti

Università di Ferrara, Dipartimento di Fisica and INFN, I-44100 Ferrara, Italy

E. Treadwell

Florida AEM University, Tallahassee, FL 3230\%, USA

F. Anulli, R. Baldini-Ferroli, A. Calcaterra, R. de Sangro, G. Finocchiaro, P. Patteri, I. M. Peruzzi, M. Piccolo, A. Zallo

Laboratori Nazionali di Frascati dell'INFN, I-00044 Frascati, Italy

A. Buzzo, R. Capra, R. Contri, G. Crosetti, M. Lo Vetere, M. Macri, M. R. Monge, S. Passaggio,

C. Patrignani, E. Robutti, A. Santroni, S. Tosi

Università di Genova, Dipartimento di Fisica and INFN, I-16146 Genova, Italy

S. Bailey, G. Brandenburg, K. S. Chaisanguanthum, M. Morii, E. Won

Harvard University, Cambridge, MA 02138, USA 
R. S. Dubitzky, U. Langenegger

Universität Heidelberg, Physikalisches Institut, Philosophenweg 12, D-69120 Heidelberg, Germany

W. Bhimji, D. A. Bowerman, P. D. Dauncey, U. Egede, J. R. Gaillard, G. W. Morton, J. A. Nash, M. B. Nikolich, G. P. Taylor Imperial College London, London, SWr 2AZ, United Kingdom

M. J. Charles, G. J. Grenier, U. Mallik University of Iowa, Iowa City, IA 52242, USA

J. Cochran, H. B. Crawley, J. Lamsa, W. T. Meyer, S. Prell, E. I. Rosenberg, A. E. Rubin, J. Yi Iowa State University, Ames, IA 50011-3160, USA

M. Biasini, R. Covarelli, M. Pioppi

Università di Perugia, Dipartimento di Fisica and INFN, I-06100 Perugia, Italy

M. Davier, X. Giroux, G. Grosdidier, A. Höcker, S. Laplace, F. Le Diberder, V. Lepeltier, A. M. Lutz, T. C. Petersen, S. Plaszczynski, M. H. Schune, L. Tantot, G. Wormser

Laboratoire de l'Accélérateur Linéaire, F-91898 Orsay, France

C. H. Cheng, D. J. Lange, M. C. Simani, D. M. Wright

Lawrence Livermore National Laboratory, Livermore, CA 94550, USA

A. J. Bevan, C. A. Chavez, J. P. Coleman, I. J. Forster, J. R. Fry, E. Gabathuler, R. Gamet, D. E. Hutchcroft, R. J. Parry, D. J. Payne, R. J. Sloane, C. Touramanis

University of Liverpool, Liverpool L69 72E, United Kingdom

J. J. Back, ${ }^{1}$ C. M. Cormack, P. F. Harrison, ${ }^{1}$ F. Di Lodovico, G. B. Mohanty ${ }^{1}$

Queen Mary, University of London, E1 4 NS, United Kingdom

C. L. Brown, G. Cowan, R. L. Flack, H. U. Flaecher, M. G. Green, P. S. Jackson, T. R. McMahon, S. Ricciardi, F. Salvatore, M. A. Winter

University of London, Royal Holloway and Bedford New College, Egham, Surrey TW20 0EX, United Kingdom

D. Brown, C. L. Davis

University of Louisville, Louisville, KY 40292, USA

J. Allison, N. R. Barlow, R. J. Barlow, P. A. Hart, M. C. Hodgkinson, G. D. Lafferty, A. J. Lyon, J. C. Williams

University of Manchester, Manchester M13 9PL, United Kingdom

A. Farbin, W. D. Hulsbergen, A. Jawahery, D. Kovalskyi, C. K. Lae, V. Lillard, D. A. Roberts

University of Maryland, College Park, MD 20742, USA

G. Blaylock, C. Dallapiccola, K. T. Flood, S. S. Hertzbach, R. Kofler, V. B. Koptchev, T. B. Moore, S. Saremi, H. Staengle, S. Willocq

University of Massachusetts, Amherst, MA 01003, USA

\footnotetext{
${ }^{1}$ Now at Department of Physics, University of Warwick, Coventry, United Kingdom
} 
R. Cowan, G. Sciolla, S. J. Sekula, F. Taylor, R. K. Yamamoto

Massachusetts Institute of Technology, Laboratory for Nuclear Science, Cambridge, MA 02139, USA

D. J. J. Mangeol, P. M. Patel, S. H. Robertson

McGill University, Montréal, QC, Canada H3A $2 T 8$

A. Lazzaro, V. Lombardo, F. Palombo

Università di Milano, Dipartimento di Fisica and INFN, I-20133 Milano, Italy

J. M. Bauer, L. Cremaldi, V. Eschenburg, R. Godang, R. Kroeger, J. Reidy, D. A. Sanders, D. J. Summers, H. W. Zhao

University of Mississippi, University, MS 38677, USA

S. Brunet, D. Côté, P. Taras

Université de Montréal, Laboratoire René J. A. Lévesque, Montréal, QC, Canada H3C 3J7

H. Nicholson

Mount Holyoke College, South Hadley, MA 01075, USA

N. Cavallo, F. Fabozzi, ${ }^{2}$ C. Gatto, L. Lista, D. Monorchio, P. Paolucci, D. Piccolo, C. Sciacca

Università di Napoli Federico II, Dipartimento di Scienze Fisiche and INFN, I-80126, Napoli, Italy

M. Baak, H. Bulten, G. Raven, H. L. Snoek, L. Wilden

NIKHEF, National Institute for Nuclear Physics and High Energy Physics, NL-1009 DB Amsterdam, The Netherlands

C. P. Jessop, J. M. LoSecco

University of Notre Dame, Notre Dame, IN 46556, USA

T. Allmendinger, K. K. Gan, K. Honscheid, D. Hufnagel, H. Kagan, R. Kass, T. Pulliam, A. M. Rahimi, R. Ter-Antonyan, Q. K. Wong

Ohio State University, Columbus, OH 43210, USA

J. Brau, R. Frey, O. Igonkina, C. T. Potter, N. B. Sinev, D. Strom, E. Torrence

University of Oregon, Eugene, OR 97403, USA

F. Colecchia, A. Dorigo, F. Galeazzi, M. Margoni, M. Morandin, M. Posocco, M. Rotondo, F. Simonetto, R. Stroili, G. Tiozzo, C. Voci

Università di Padova, Dipartimento di Fisica and INFN, I-35131 Padova, Italy

M. Benayoun, H. Briand, J. Chauveau, P. David, Ch. de la Vaissière, L. Del Buono, O. Hamon, M. J. J. John, Ph. Leruste, J. Malcles, J. Ocariz, M. Pivk, L. Roos, S. T'Jampens, G. Therin Universités Paris VI et VII, Laboratoire de Physique Nucléaire et de Hautes Energies, F-75252 Paris, France

P. F. Manfredi, V. Re

Università di Pavia, Dipartimento di Elettronica and INFN, I-27100 Pavia, Italy

\footnotetext{
${ }^{2}$ Also with Università della Basilicata, Potenza, Italy
} 


\author{
P. K. Behera, L. Gladney, Q. H. Guo, J. Panetta \\ University of Pennsylvania, Philadelphia, PA 19104, USA
}

C. Angelini, G. Batignani, S. Bettarini, M. Bondioli, F. Bucci, G. Calderini, M. Carpinelli, F. Forti, M. A. Giorgi, A. Lusiani, G. Marchiori, F. Martinez-Vidal, ${ }^{3}$ M. Morganti, N. Neri, E. Paoloni, M. Rama, G. Rizzo, F. Sandrelli, J. Walsh

Università di Pisa, Dipartimento di Fisica, Scuola Normale Superiore and INFN, I-56127 Pisa, Italy

M. Haire, D. Judd, K. Paick, D. E. Wagoner

Prairie View A\&M University, Prairie View, TX 7r446, USA

N. Danielson, P. Elmer, Y. P. Lau, C. Lu, V. Miftakov, J. Olsen, A. J. S. Smith, A. V. Telnov

Princeton University, Princeton, NJ 08544, USA

F. Bellini, G. Cavoto, ${ }^{4}$ R. Faccini, F. Ferrarotto, F. Ferroni, M. Gaspero, L. Li Gioi, M. A. Mazzoni, S. Morganti, M. Pierini, G. Piredda, F. Safai Tehrani, C. Voena Università di Roma La Sapienza, Dipartimento di Fisica and INFN, I-00185 Roma, Italy

S. Christ, G. Wagner, R. Waldi

Universität Rostock, D-18051 Rostock, Germany

T. Adye, N. De Groot, B. Franek, N. I. Geddes, G. P. Gopal, E. O. Olaiya

Rutherford Appleton Laboratory, Chilton, Didcot, Oxon, OX11 0QX, United Kingdom

R. Aleksan, S. Emery, A. Gaidot, S. F. Ganzhur, P.-F. Giraud, G. Hamel de Monchenault, W. Kozanecki, M. Legendre, G. W. London, B. Mayer, G. Schott, G. Vasseur, Ch. Yèche, M. Zito

DSM/Dapnia, CEA/Saclay, F-91191 Gif-sur-Yvette, France

M. V. Purohit, A. W. Weidemann, J. R. Wilson, F. X. Yumiceva

University of South Carolina, Columbia, SC 29208, USA

D. Aston, R. Bartoldus, N. Berger, A. M. Boyarski, O. L. Buchmueller, R. Claus, M. R. Convery, M. Cristinziani, G. De Nardo, D. Dong, J. Dorfan, D. Dujmic, W. Dunwoodie, E. E. Elsen, S. Fan,

R. C. Field, T. Glanzman, S. J. Gowdy, T. Hadig, V. Halyo, C. Hast, T. Hryn'ova, W. R. Innes,

M. H. Kelsey, P. Kim, M. L. Kocian, D. W. G. S. Leith, J. Libby, S. Luitz, V. Luth, H. L. Lynch,

H. Marsiske, R. Messner, D. R. Muller, C. P. O'Grady, V. E. Ozcan, A. Perazzo, M. Perl, S. Petrak,

B. N. Ratcliff, A. Roodman, A. A. Salnikov, R. H. Schindler, J. Schwiening, G. Simi, A. Snyder, A. Soha, J. Stelzer, D. Su, M. K. Sullivan, J. Va'vra, S. R. Wagner, M. Weaver, A. J. R. Weinstein,

W. J. Wisniewski, M. Wittgen, D. H. Wright, A. K. Yarritu, C. C. Young

Stanford Linear Accelerator Center, Stanford, CA 94309, USA

P. R. Burchat, A. J. Edwards, T. I. Meyer, B. A. Petersen, C. Roat

Stanford University, Stanford, CA 94305-4060, USA

S. Ahmed, M. S. Alam, J. A. Ernst, M. A. Saeed, M. Saleem, F. R. Wappler State University of New York, Albany, NY 12222, USA

\footnotetext{
${ }^{3}$ Also with IFIC, Instituto de Física Corpuscular, CSIC-Universidad de Valencia, Valencia, Spain

${ }^{4}$ Also with Princeton University, Princeton, USA
} 
W. Bugg, M. Krishnamurthy, S. M. Spanier

University of Tennessee, Knoxville, TN 37996, USA

R. Eckmann, H. Kim, J. L. Ritchie, A. Satpathy, R. F. Schwitters

University of Texas at Austin, Austin, TX 78712, USA

J. M. Izen, I. Kitayama, X. C. Lou, S. Ye

University of Texas at Dallas, Richardson, TX 75083, USA

F. Bianchi, M. Bona, F. Gallo, D. Gamba

Università di Torino, Dipartimento di Fisica Sperimentale and INFN, I-10125 Torino, Italy

L. Bosisio, C. Cartaro, F. Cossutti, G. Della Ricca, S. Dittongo, S. Grancagnolo, L. Lanceri, P. Poropat, ${ }^{5}$ L. Vitale, G. Vuagnin

Università di Trieste, Dipartimento di Fisica and INFN, I-34127 Trieste, Italy

R. S. Panvini

Vanderbilt University, Nashville, TN 37235, USA

Sw. Banerjee, C. M. Brown, D. Fortin, P. D. Jackson, R. Kowalewski, J. M. Roney, R. J. Sobie University of Victoria, Victoria, BC, Canada V8W $3 P 6$

H. R. Band, B. Cheng, S. Dasu, M. Datta, A. M. Eichenbaum, M. Graham, J. J. Hollar, J. R. Johnson, P. E. Kutter, H. Li, R. Liu, A. Mihalyi, A. K. Mohapatra, Y. Pan, R. Prepost, P. Tan, J. H. von Wimmersperg-Toeller, J. Wu, S. L. Wu, Z. Yu

University of Wisconsin, Madison, WI 53706, USA

M. G. Greene, H. Neal

Yale University, New Haven, CT 06511, USA

\footnotetext{
${ }^{5}$ Deceased
} 


\section{INTRODUCTION}

Isospin violation in decays of $e^{+} e^{-} \rightarrow B^{0} \bar{B}^{0}$ at the $\Upsilon(4 S)$ resonance results in a difference between the branching fractions $f_{00} \equiv \mathcal{B}\left(e^{+} e^{-} \rightarrow B^{0} \bar{B}^{0}\right)$ and $f_{+-} \equiv \mathcal{B}\left(e^{+} e^{-} \rightarrow B^{+} B^{-}\right)$. Measurements of the ratio $R^{+/ 0} \equiv f_{+-} / f_{00}$, summarized in Table 1, are consistent with unity within the errors [1]. Theoretical predictions for $R^{+/ 0}$ range from 1.03 to 1.25 2]. Currently, almost all published measurements of $B$ meson branching fractions make the assumption that $R^{+/ 0}=1$. Precision measurements of $f_{00}, f_{+-}$, and $R^{+/ 0}$ can be used to eliminate this assumption and re-normalize all $B$ meson branching fractions.

Table 1: Summary of previous measurements of $R^{+/ 0}$

\begin{tabular}{lccc}
\hline \hline Decay $B \rightarrow$ & $\int \mathcal{L} d t$ & $R^{+/ 0}$ & Source \\
\hline$J / \psi\left(K^{+} / K_{s}^{0}\right)$ & $81.9 \mathrm{fb}^{-1}$ & $1.006 \pm 0.036 \pm 0.031$ & BABAR [3] \\
$D^{*(+/ 0)} \ell \bar{\nu}$ & $2.73 \mathrm{fb}^{-1}$ & $1.058 \pm 0.084 \pm 0.136$ & CLEO [4] \\
$J / \psi h^{(+/ 0)}$ & $20.7 \mathrm{fb}^{-1}$ & $1.10 \pm 0.06 \pm 0.05$ & BABAR []] \\
$J / \psi K^{*(+/ 0)}$ & $9.2 \mathrm{fb}^{-1}$ & $1.04 \pm 0.07 \pm 0.04$ & CLEO [6] \\
\hline \hline
\end{tabular}

In this paper we report the first direct measurement of $f_{00}$. The measurement is based on partial reconstruction of the decay $\bar{B}^{0} \rightarrow D^{*+} \ell^{-} \bar{\nu}_{\ell}$ (the inclusion of charge-conjugate states is implied throughout this paper). This allows a sizeable sample of double tagged events to be identified. Comparison of the double-tag and the single-tag yields allows a determination of $f_{00}$ with minimal input from simulation.

The technique used to measure $f_{00}$ is as follows: in every event we reconstruct the decay $\bar{B}^{0} \rightarrow$ $D^{*+} \ell^{-} \bar{\nu}_{\ell}$, as described further below. The sample of events in which at least one $\bar{B}^{0} \rightarrow D^{*+} \ell^{-} \bar{\nu}_{\ell}$ candidate decay is found is labeled as "single-tag sample". The number of signal decays found in this sample is

$$
N_{s}=2 N_{B \bar{B}} f_{00} \epsilon_{s} \mathcal{B}\left(\bar{B}^{0} \rightarrow D^{*+} \ell^{-} \bar{\nu}_{\ell}\right),
$$

where $N_{B \bar{B}}=(88726 \pm 23) \times 10^{3}$ is the total number of $B \bar{B}$ events in the data sample and $\epsilon_{s}$ is the reconstruction efficiency of the decay $\bar{B}^{0} \rightarrow D^{*+} \ell^{-} \bar{\nu}_{\ell}$. The technique for measuring $N_{B \bar{B}}$ is described in [7]. The data sample has a mean energy of $10.580 \mathrm{GeV}[8]$ and an energy spread of only $4.6 \mathrm{MeV}$. Such a small spread means that any energy dependence of $f_{00}$ has a negligible effect on the central value. The subset of single-tag events in which two $\bar{B}^{0} \rightarrow D^{*+} \ell^{-} \bar{\nu}_{\ell}$ candidates are found is labeled as "double-tag sample". The number of such events is

$$
N_{d}=N_{B \bar{B}} f_{00} \epsilon_{d}\left[\mathcal{B}\left(\bar{B}^{0} \rightarrow D^{*+} \ell^{-} \bar{\nu}_{\ell}\right)\right]^{2},
$$

where $\epsilon_{d}$ is the efficiency to reconstruct two $\bar{B}^{0} \rightarrow D^{*+} \ell^{-} \bar{\nu}_{\ell}$ decays in the same event. Note that every double-tag event contributes two entries to the single-tag sample. Using Eq. (11) and Eq. (2), the ratio $f_{00}$ is given by

$$
f_{00}=\frac{C N_{s}^{2}}{4 N_{d} N_{B \bar{B}}}
$$

where we have defined the coefficient $C \equiv \epsilon_{d} / \epsilon_{s}^{2} . \quad C=1$ if the efficiencies for detecting each $B$ meson are uncorrelated in double-tag events. 


\section{THE BABAR DETECTOR AND DATASET}

The BABAR data sample used in this paper consists of $81.7 \mathrm{fb}^{-1}$ collected at the $\Upsilon(4 S)$ resonance (the on-resonance sample) and $9.6 \mathrm{fb}^{-1}$ collected $40 \mathrm{MeV}$ below the resonance (the off-resonance sample). Simulated $B \bar{B}$ events were analyzed through the same analysis chain as the data. The equivalent luminosity of the simulated sample is approximately three times that of the on-resonance data.

A detailed description of the BABAR detector and the algorithms used for track reconstruction and particle identification is provided elsewhere [9. A brief summary is given here. Highmomentum particles are reconstructed by matching hits in the silicon vertex tracker (SVT) with track elements in the drift chamber (DCH). Lower momentum tracks, which do not leave signals on many wires in the $\mathrm{DCH}$ due to the bending induced by a magnetic field, are reconstructed by the SVT alone. Electrons are identified with the ratio of the track momentum to the associated energy deposited in the calorimeter (EMC), the transverse profile of the shower, the energy loss in the drift chamber, and the information from a Cherenkov detector (DIRC). Muons are identified in the instrumented flux return (IFR), composed of resistive plate chambers and layers of iron. Muon candidates are required to have a path length and hit distribution in the instrumented flux return and energy deposition in the EMC consistent with that expected for a minimum-ionizing particle. The Cherenkov light emission in the DIRC is then employed to further reject kaons misidentified as muons by requiring muon candidates to have a kaon hypothesis probability less than $5 \%$.

Hadronic events are selected by requiring at least four charged particle tracks reconstructed by the silicon vertex detector and the drift chamber. To reduce background from continuum $e^{+} e^{-} \rightarrow q \bar{q}$, where $q$ stands for a $u, d, s$, or $c$ quark, the ratio $R_{2}=H_{2} / H_{0}$ of the second to the zeroth Fox-Wolfram moments is used [10.

\section{ANALYSIS METHOD}

We reconstruct the decays $\bar{B}^{0} \rightarrow D^{*+} \ell^{-} \bar{\nu}_{\ell}$ with a partial reconstruction technique. The application of the technique to this mode was first proposed by the ARGUS Collaboration [1] and has been used by CLEO [4, DELPHI [12, OPAL [13, and BABAR [14. In this technique, only the lepton from the decay $\bar{B}^{0} \rightarrow D^{*+} \ell^{-} \bar{\nu}_{\ell}$ and the soft pion from the decay $D^{*+} \rightarrow D^{0} \pi^{+}$are used. No attempt is made to reconstruct the $D^{0}$, resulting in high reconstruction efficiency.

To suppress leptons from charm decays, all lepton candidates (electrons and muons) are required to have momentum between $1.5 \mathrm{GeV} / c$ and $2.5 \mathrm{GeV} / c$ in the $e^{+} e^{-}$center-of-mass $(\mathrm{CM})$ frame. Soft pion candidates are required to have CM momentum between $60 \mathrm{MeV} / c$ and $200 \mathrm{MeV} / c$. As a consequence of the limited phase space available in the $D^{*+}$ decay, the soft pion is emitted within a one radian-wide cone centered about the $D^{*+}$ direction in the CM frame. The $D^{*+}$ fourmomentum can therefore be computed by approximating its direction as that of the soft pion, and parameterizing its momentum as a linear function of the soft pion momentum, with parameters obtained from the simulation. The presence of an undetected neutrino is inferred from conservation of momentum and energy. The neutrino invariant mass squared is calculated:

$$
\mathcal{M}^{2} \equiv\left(E_{\text {beam }}-E_{D^{*}}-E_{\ell}\right)^{2}-\left(\mathbf{p}_{D^{*}}+\mathbf{p}_{\ell}\right)^{2},
$$

where $E_{\text {beam }}$ is the beam energy and $E_{\ell}\left(E_{D^{*}}\right)$ and $\mathbf{p}_{\ell}\left(\mathbf{p}_{D^{*}}\right)$ are the CM energy and momentum of the lepton (the $D^{*}$ meson). If the decay is properly reconstructed and the neutrino is the only 
missing particle, the $\mathcal{M}^{2}$ distribution will peak near zero for signal events. Background events, however, are spread over a wide range of $\mathcal{M}^{2}$ values.

In what follows, we use the symbol $\mathcal{M}_{s}^{2}$ to denote $\mathcal{M}^{2}$ for any candidate in the single-tag sample. In the double-tag sample, we randomly choose one of the two reconstructed $\bar{B}^{0} \rightarrow D^{*+} \ell^{-} \nu_{l}$ candidates as "first" and the other as "second". Their $\mathcal{M}^{2}$ values are labeled $\mathcal{M}_{1}^{2}$ and $\mathcal{M}_{2}^{2}$, respectively. For each of the variables $\mathcal{M}_{i}^{2}(i=s, 1,2)$, we define a signal region $\mathcal{M}_{i}^{2}>-2 \mathrm{GeV}^{2} / c^{4}$ and the sideband $-8<\mathcal{M}_{i}^{2}<-4 \mathrm{GeV}^{2} / c^{4}$.

In addition to signal $\bar{B}^{0} \rightarrow D^{*+} \ell^{-} \nu_{l}$ decays, the single-tag and double-tag samples contain several types of events:

- Continuum $e^{+} e^{-} \rightarrow q \bar{q}$ background.

- Combinatorial $B \bar{B}$ background, formed from random combinations of reconstructed leptons and soft pions. This background can also be due to the low momentum soft pions not coming from a $D^{*}$, produced by either the same $B$ or other $B$ [15].

- Peaking $B \bar{B}$ background, composed of $\bar{B} \rightarrow D^{*}(n \pi) \ell \bar{\nu}_{\ell}$ decays with or without an excited charmed resonance $\left(D^{* *}\right)$ [16], where the reconstructed soft pion comes from the decay $D^{*+} \rightarrow$ $D^{0} \pi^{+}$, leading to an accumulation of these events at high values of $\mathcal{M}_{i}^{2}$. These events are peaking background and are produced both by $\bar{B}^{0}$ and $B^{-}$decays. Their $\mathcal{M}_{i}^{2}$ distribution differs from the signal, which allows us to extract their contribution in a fit. Such events are suppressed by the requirement $p_{\ell}>1.5 \mathrm{GeV} / c$ on the lepton $\mathrm{CM}$ momentum.

- The decays $\bar{B}^{0} \rightarrow D^{*+} \tau^{-} \bar{\nu}_{\tau}$ and $\bar{B}^{0} \rightarrow D^{*+} \ell^{-} \bar{\nu}_{\ell}(n \gamma)$ may be used for the measurement of $f_{00}$ and are therefore considered as signal. These events peak in $\mathcal{M}_{i}^{2}$ and come from $\bar{B}^{0}$ decays.

The double-tag sample contains two additional types of background: $\mathcal{M}_{1}^{2}$-combinatorial and $\mathcal{M}_{1}^{2}$ peaking. In $\mathcal{M}_{1}^{2}$-combinatorial $\left(\mathcal{M}_{1}^{2}\right.$-peaking) background events the first candidate is combinatorial (peaking) background.

To determine $N_{s}$ and $N_{d}$, we perform binned $\chi^{2}$ fits to one-dimensional histograms of the $\mathcal{M}_{s}^{2}$ and $\mathcal{M}_{2}^{2}$ distributions of on-resonance data events, ranging from -8 to $2 \mathrm{GeV}^{2} / c^{4}$. Before fitting, we subtract the continuum background contribution from the histograms. This is done using the $\mathcal{M}_{s}^{2}$ and $\mathcal{M}_{2}^{2}$ distributions of off-resonance data, scaled to account for the ratio of on-resonance to off-resonance luminosities and the CM energy dependence of the continuum production crosssection. In addition, the contributions of the $\mathcal{M}_{1}^{2}$-combinatorial and $\mathcal{M}_{1}^{2}$-peaking backgrounds are subtracted from the $\mathcal{M}_{2}^{2}$ histogram before the fit. The $\mathcal{M}_{1}^{2}$-combinatorial background is determined from the $\mathcal{M}_{1}^{2}$ sideband, which contain only continuum and combinatorial background events. This histogram is scaled by the ratio of the number of combinatorial events in the signal region and the sideband, determined from the simulation. The $\mathcal{M}_{1}^{2}$-peaking background subtraction is based on the simulated $\mathcal{M}_{1}^{2}$-peaking events.

After the subtraction, the $\mathcal{M}_{s}^{2}$ and $\mathcal{M}_{2}^{2}$ histograms are fit separately, using a function whose value for bin $j$ of the histogram is

$$
f_{j}=\sum_{t} N^{t} P_{j}^{t}
$$

where $N^{t}$ is the number of events of type $t$ ( $t=$ signal, combinatorial, peaking) populating the histogram, and $P_{j}^{t}$ is the bin $j$ value of a discrete probability density function (PDF) obtained from simulated events of type $t$, normalized such that $\sum_{j} P_{j}^{t}=1$. The fit determines the parameters $N^{t}$ 
by minimizing:

$$
\chi^{2}=\sum_{j} \frac{\left(H_{j}-f_{j}\right)^{2}}{\sigma_{H_{j}}^{2}+\sigma_{f_{j}}^{2}},
$$

where $H_{j}$ is the number of entries in bin $j$ of the histogram being fit; $\sigma_{H_{j}}$ is the statistical error on $H_{j}$, including uncertainties due to the background subtractions described above; and $\sigma_{f_{j}}$ is the error on $f_{j}$, determined from the errors on $P_{j}^{t}$, which are due to the finite size of the simulated sample.

The results of the fits are presented in Table 2. The $\mathcal{M}_{s}^{2}$ and $\mathcal{M}_{2}^{2}$ distributions are shown in Fig. 11 with the contributions of the different event types indicated. The fits yield the values $N_{s}=$ $786300 \pm 1950$ and $N_{d}=3560 \pm 80$. Using the simulation we determine $C=0.9946 \pm 0.0078$, where the error is due to the finite size of the simulated sample. Eq. (3) then gives $f_{00}=0.486 \pm 0.010$, where the error is due to data statistics only.

Table 2: Numbers of entries of different types found by the fits to the $\mathcal{M}_{s}^{2}$ and $\mathcal{M}_{2}^{2}$ histograms in the signal region. Also shown are the numbers of entries of subtracted backgrounds and the confidence levels of the fits.

\begin{tabular}{lcc}
\hline \hline Source & $\mathcal{M}_{s}^{2}$ & $\mathcal{M}_{2}^{2}$ \\
\hline Combinatorial $B \bar{B}$ & $558090 \pm 760$ & $1520 \pm 40$ \\
Peaking $B \bar{B}$ & $68170 \pm 260$ & $300 \pm 20$ \\
Signal & $786300 \pm 2000$ & $3560 \pm 80$ \\
\hline Continuum & $238500 \pm 1300$ & $160 \pm 40$ \\
$\mathcal{M}_{1}^{2}$-combinatorial & - & $180 \pm 20$ \\
$\mathcal{M}_{1}^{2}$-peaking & - & $60 \pm 10$ \\
\hline$\chi^{2} /$ d.o.f. & $41 / 56$ & $48 / 56$ \\
Confidence level & $93 \%$ & $77 \%$ \\
\hline \hline
\end{tabular}

To determine how well the simulation reproduces the $\mathcal{M}_{s}^{2}$ and $\mathcal{M}_{2}^{2}$ distributions of the combinatorial background in the data, we study the distributions of a sample of same-charge candidates, in which the lepton and soft pion have the same electric charge. This sample contains only continuum and combinatorial $B \bar{B}$ background. We fit the continuum-subtracted $\mathcal{M}_{s}^{2}$ and $\mathcal{M}_{2}^{2}$ histograms of the same-charge sample using the function $f_{j}^{\prime}=N P_{j}^{\prime}$, where $P_{j}^{\prime}$ is the bin $j$ value of the PDF of same-charge simulated $B \bar{B}$ events, normalized such that $\sum_{j} P_{j}^{\prime}=1$, and the parameter $N$ is determined by the fit. The histograms, overlaid with the fit function, are shown in Fig. 2. The ratio between these two histograms is fitted to a constant both for the $\mathcal{M}_{s}^{2}$ and $\mathcal{M}_{2}^{2}$ summed over the signal region and over all bins are shown in Fig. 3. The accumulated differences $D \equiv \sum_{j}\left(H_{j}^{\prime}-f_{j}^{\prime}\right)$ between the same-charge data histograms $H_{j}^{\prime}$ and the fit functions are summarized in Table 3. Their consistency with zero indicates that the distributions of simulated combinatorial $B \bar{B}$ background events do not lead to significant fake signal yields.

\section{SYSTEMATIC STUDIES}

We consider several sources of systematic uncertainties in $f_{00}$. All estimated errors are an absolute systematic uncertainties in $f_{00}$ and summarized in Table 4. 
Table 3: The difference $D \equiv \sum_{j}\left(H_{j}^{\prime}-f_{j}^{\prime}\right)$ between the same-charge data histogram and the fit function, summed over all bins or over the signal region only. Also shown are the fit $\chi^{2} /$ d.o.f. values and confidence levels.

\begin{tabular}{l|cc|cc}
\hline \hline & \multicolumn{2}{|c|}{ Signal region } & \multicolumn{2}{c}{ All bins } \\
& $\mathcal{M}_{s}^{2}$ & $\mathcal{M}_{2}^{2}$ & $\mathcal{M}_{s}^{2}$ & $\mathcal{M}_{2}^{2}$ \\
\hline$D$ & $-1300 \pm 2100$ & $-80 \pm 80$ & $-700 \pm 3000$ & $-70 \pm 80$ \\
$\chi^{2} /$ d.o.f. & $17 / 19$ & $13 / 19$ & $40 / 55$ & $34 / 53$ \\
C.L.(\%) & 59 & 84 & 94 & 98 \\
\hline \hline
\end{tabular}

Table 4: Summary of the absolute systematic errors for $f_{00}$.

\begin{tabular}{lc}
\hline \hline Source & $\delta\left(f_{00}\right)$ \\
\hline $\mathcal{M}_{1}^{2}$-combinatorial & 0.0005 \\
$\mathcal{M}_{1}^{2}$-peaking & 0.0005 \\
Same charged events & 0.0025 \\
Peaking background & 0.004 \\
$B$-meson counting & 0.0055 \\
$\Upsilon(4 S) \rightarrow$ non- $B \bar{B}$ & 0.0025 \\
Efficiency correlation & 0.004 \\
Monte Carlo statistics & 0.002 \\
\hline Total & 0.009 \\
\hline \hline
\end{tabular}

1. The systematic uncertainty from the $\mathcal{M}_{1}^{2}$-combinatorial contribution subtraction in the $\mathcal{M}_{2}^{2}$ histogram is 0.0005 . The error is obtained by varying the total $\mathcal{M}_{1}^{2}$-combinatorial background by its statistical error and repeating the analysis.

2. An error of 0.0005 is estimated due to the subtraction of the $\mathcal{M}_{1}^{2}$-peaking contribution in the $\mathcal{M}_{2}^{2}$ histogram. The error is obtained by comparing the ratio between the numbers of subtracted $\mathcal{M}_{1}^{2}$-peaking and $\mathcal{M}_{1}^{2}$-combinatorial events with their ratio of peaking and combinatorial events in Table 2

3. Propagating the errors on the quantities $D$ (same charged events) of Table 3 leads to an error of 0.0025 on $f_{00}$. To determine this error we vary the signal events both for the single-tag and the double-tag samples. The largest uncertainty then is taken for the uncertainty on $f_{00}$.

4. The PDFs $P^{t}(t=$ peak) of the peaking background come from simulated event samples containing different $D^{* *}$ resonances or non-resonant events. We vary the ratio of the branching fraction of the resonant and the non-resonant production such that the variation of this ratio is wide enough to include poorly known decays. We repeat the analysis procedure to determine $N_{s}$ and $N_{d}$. The resulting error on $f_{00}$ is 0.004 .

5. Uncertainties in the branching fractions of $\bar{B}^{0} \rightarrow D^{*+} \tau^{-} \bar{\nu}_{\tau}$ and $\bar{B}^{0} \rightarrow D^{*+} \ell^{-} \bar{\nu}_{\ell}(n \gamma)$ relative to $\bar{B}^{0} \rightarrow D^{*+} \ell^{-} \bar{\nu}_{\ell}$ lead to uncertainties in the PDFs $P^{t}(t=$ signal $)$ of the signal events. This uncertainty in $f_{00}$ is negligible. 
6. The error due to the uncertainty in $N_{B \bar{B}}$ is 0.0055 . It includes the uncertainties for differences in the cross sections and efficiencies for muon pairs and continuum events between on-resonance and off-resonance samples, hadronic selection criteria and the uncertainties in the tracking efficiency.

7. In this paper the impact of non- $B \bar{B}$ decays of the $\Upsilon(4 S)$ on $B$-meson counting has been accounted for as a systematic error. The upper limit for the branching fraction of $\Upsilon(4 S)$ decays into non- $B \bar{B}$ is $4 \%$ at $95 \%$ confidence level [17]. We conservatively estimate the systematic error by decreasing the $4 \%$ upper limit on the branching fraction to $2 \%$. From this variation we estimate an error of 0.0025 due to the effect on $N_{B \bar{B}}$ of a possible decay.

8. We note that the lepton momentum spectrum in the Monte Carlo simulation is different from the one we observe in the data. We tune the simulation to the data by rejecting simulated events in such a way that the two lepton momentum spectra agree. We repeat the analysis procedure without the rejected events. The systematic error due to the uncertainty in the lepton momentum spectrum is negligible.

9. There is a small efficiency correlation between the single-tag and the double-tag samples. The systematic uncertainty due to this efficiency correlation is estimated by propagating the Monte Carlo simulation systematics error of $C$ into $f_{00}$. The simulation statistical error in $C$ leads to a 0.004 error in $f_{00}$. In addition to the Monte Carlo simulation systematics error of $C$, we study the effect of track multiplicity on the efficiency correlation.

10. We perform a similar procedure as mentioned above for the pion momentum spectrum. The error due to the uncertainty in the pion momentum spectrum is negligible.

11. An error of 0.002 is due to the finite size of the simulated sample, calculated using $\sigma_{f_{j}}$ in Eq. (6).

12. The $\chi^{2}$ estimator used in Eq. (6) can be biased. We did an alternative binned likelihood fit and found that the result differed by only $0.03 \%$ for $f_{00}$.

We combine the uncertainties given above in quadrature to determine an absolute systematic error of 0.009 in $f_{00}$.

\section{SUMMARY}

To summarize, using partial reconstruction of the decay $\bar{B}^{0} \rightarrow D^{*+} \ell^{-} \nu_{l}$ we have obtained a preliminary result for the branching fraction

$$
f_{00}=0.486 \pm 0.010 \pm 0.009
$$

where the first error is statistical and the second is systematic. Since this measurement is done by comparing the numbers of events with one and two reconstructed $\bar{B}^{0} \rightarrow D^{*+} \ell^{-} \nu_{l}$ decays, it does not depend on branching fractions of the $\bar{B}^{0}$ and the $D^{*+}$ decay chains, on the simulated reconstruction efficiency, on the ratio of the charged and neutral $B$ meson lifetimes, nor on assumptions of isospin symmetry. 


\section{ACKNOWLEDGMENTS}

We are grateful for the extraordinary contributions of our PEP-II colleagues in achieving the excellent luminosity and machine conditions that have made this work possible. The success of this project also relies critically on the expertise and dedication of the computing organizations that support BABAR. The collaborating institutions wish to thank SLAC for its support and the kind hospitality extended to them. This work is supported by the US Department of Energy and National Science Foundation, the Natural Sciences and Engineering Research Council (Canada), Institute of High Energy Physics (China), the Commissariat à l'Energie Atomique and Institut National de Physique Nucléaire et de Physique des Particules (France), the Bundesministerium für Bildung und Forschung and Deutsche Forschungsgemeinschaft (Germany), the Istituto Nazionale di Fisica Nucleare (Italy), the Foundation for Fundamental Research on Matter (The Netherlands), the Research Council of Norway, the Ministry of Science and Technology of the Russian Federation, and the Particle Physics and Astronomy Research Council (United Kingdom). Individuals have received support from CONACyT (Mexico), the A. P. Sloan Foundation, the Research Corporation, and the Alexander von Humboldt Foundation.

\section{References}

[1] Particle Data Group, S. Eidelman et al., Phys. Lett. B 592, 1 (2004).

[2] R. Kaiser, A. V. Manohar, and T. Mehen, Phys. Rev. Lett. 90, 142001 (2003);

M. B. Voloshin, Mod. Phys. Lett. A 18, 1783 (2003); N. Byers and E. Eichten, Phys. Rev. D 42, 3885 (1990); G. P. Lepage, Phys. Rev. D 42, 3251 (1990); D. Atwood and W. J. Marciano, Phys. Rev. D 41, 1736 (1990); E. Eichten, K. Gottfried, T. Kinoshita, and K. D. Lane, Phys. Rev. D 21, 203 (1980).

[3] BABAR Collaboration, B. Aubert et al., Phys. Rev. D 69, 071101 (2004).

[4] CLEO Collaboration, S. B. Athar et al., Phys. Rev. D 66, 052003 (2002).

[5] BABAR Collaboration, B. Aubert et al., Phys. Rev. D 65, 032001 (2002).

[6] CLEO Collaboration, J. P. Alexander et al., Phys. Rev. Lett. 86, 2737 (2001).

[7] BABAR Collaboration, B. Aubert et al., Phys. Rev. D 67, 032002 (2003).

[8] BABAR Collaboration, B. Aubert et al., hep-ex/0308020 (2003).

[9] BABAR Collaboration, B. Aubert et al., Nucl. Instrum. Meth. A 479, 1 (2002).

[10] G. C. Fox and S. Wolfram, Phys. Rev. Lett. 41, 1581 (1978).

[11] ARGUS Collaboration, H. Albrecht et al., Phys. Lett. B 324, 249 (1994).

[12] DELPHI Collaboration, P. Abreu et al., Z. Phys. C 74, 19 (1997).

[13] OPAL Collaboration, G. Abbiendi et al., Phys. Lett. B 482, 15 (2000).

[14] BABAR Collaboration, B. Aubert et al., Phys. Rev. Lett. 89, 011802 (2002). 
[15] Fermilab E791, E. M. Aitala et al., Phys. Lett. B 403, 185 (1997).

[16] Fermilab E691, J. C. Anjos et al., Phys. Rev. Lett. 62, 1717 (1989).

[17] CLEO Collaboration, B. Barish et al., Phys. Rev. Lett. 76, 1570 (1996). 

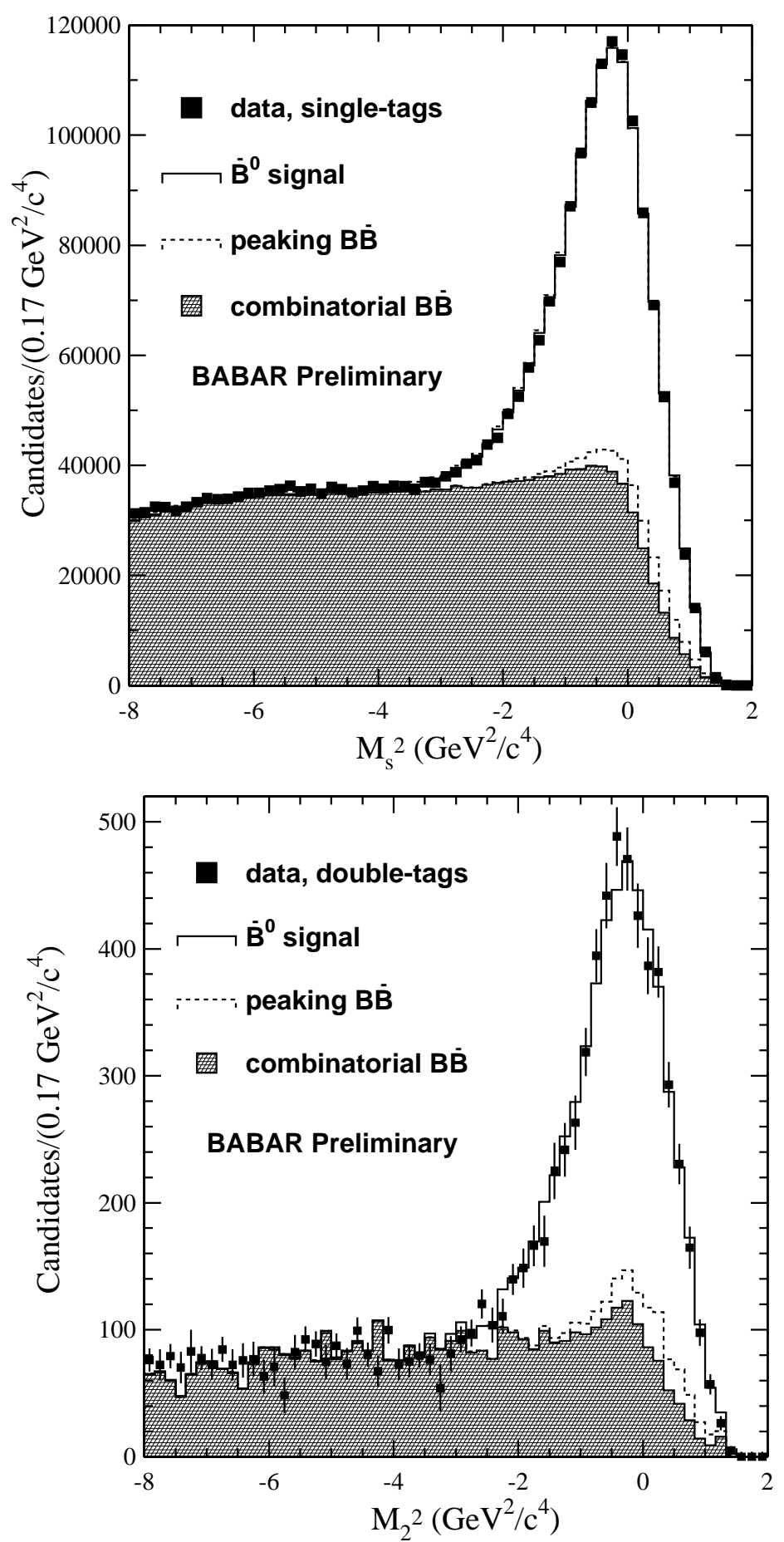

Figure 1: The $\mathcal{M}_{s}^{2}$ (top) and $\mathcal{M}_{2}^{2}$ (bottom) distributions of the on-resonance samples. The continuum background has been subtracted from the $\mathcal{M}_{s}^{2}$ distribution. For the $\mathcal{M}_{2}^{2}$ distribution, the $\mathcal{M}_{1}^{2}$-combinatorial, and the $\mathcal{M}_{1}^{2}$-peaking have been subtracted. The levels of the simulated signal, peaking $B \bar{B}$ and combinatorial $B \bar{B}$ background contributions are obtained from the fit. 


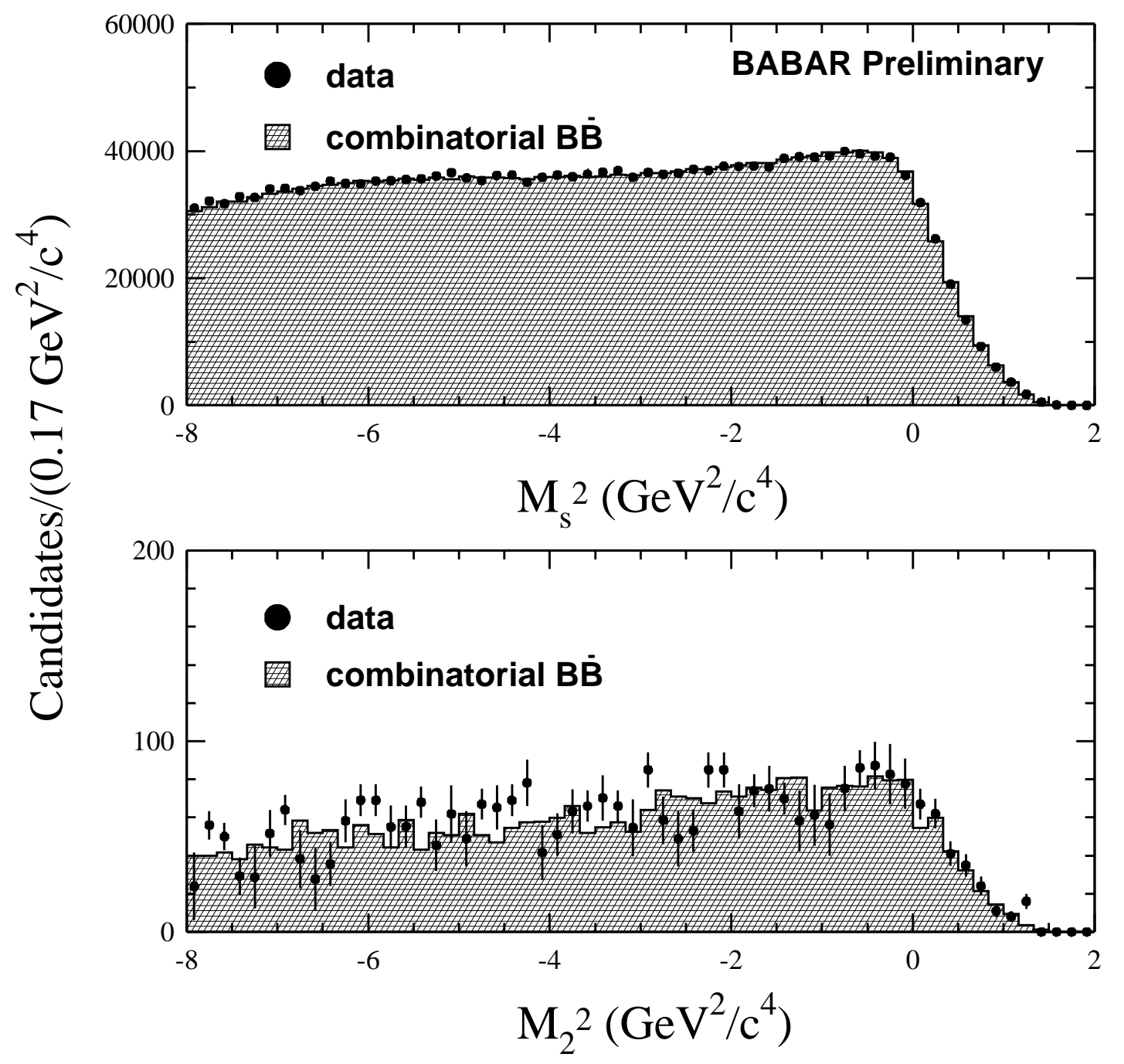

Figure 2: The $\mathcal{M}_{s}^{2}$ (top) and $\mathcal{M}_{2}^{2}$ (bottom) distributions of the same-charge on-resonance samples. The continuum background has been subtracted from the $\mathcal{M}_{s}^{2}$ distribution. For the $\mathcal{M}_{2}^{2}$ distribution, the continuum background, the $\mathcal{M}_{1}^{2}$-combinatorial and the $\mathcal{M}_{1}^{2}$-peaking backgrounds have been subtracted. The level of the simulated combinatorial $B \bar{B}$ background is obtained from the fit. 

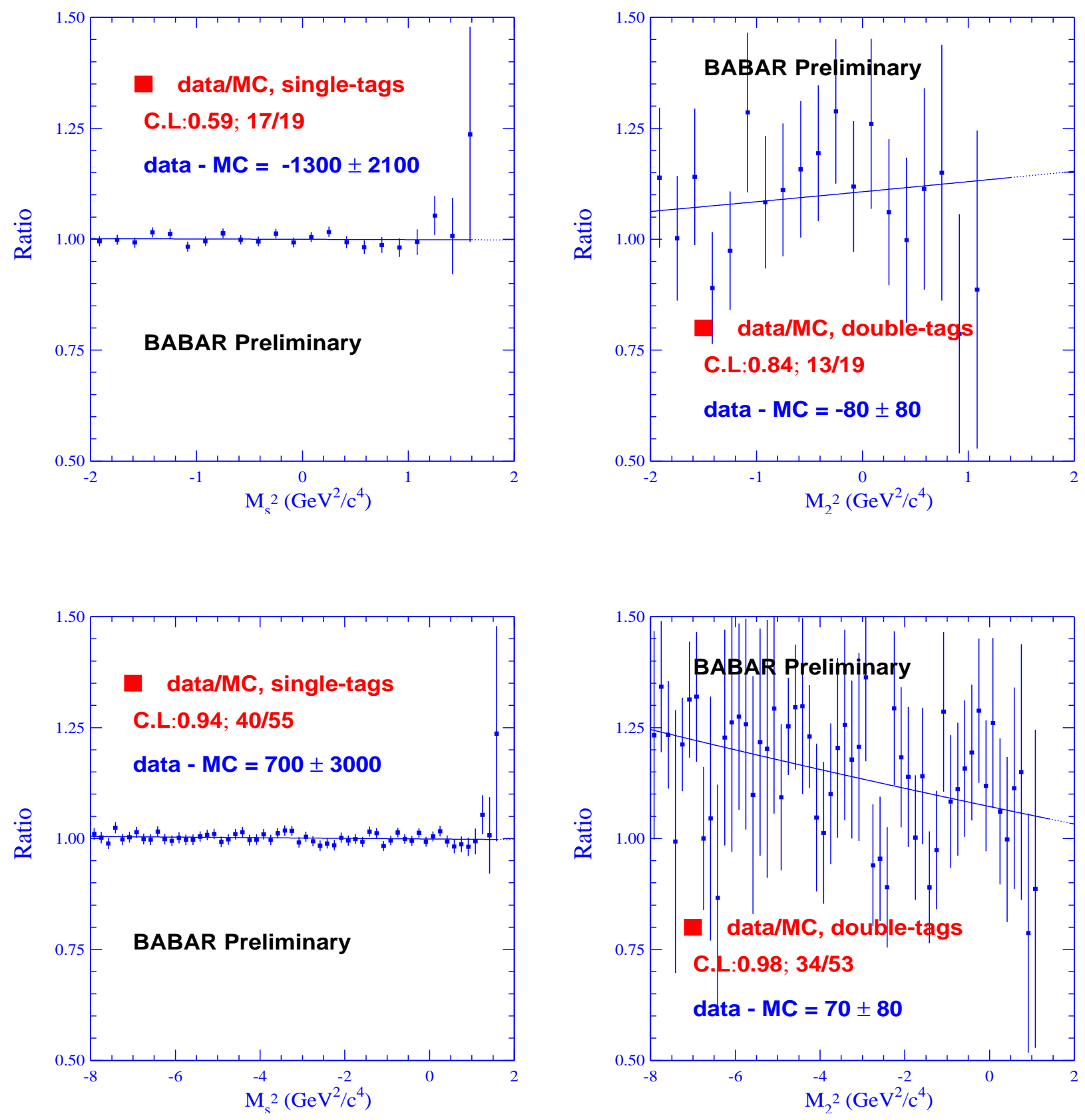

Figure 3: The ratio between data and the combinatorial $B \bar{B}$ background of the same-charge sample both for the $\mathcal{M}_{s}^{2}$ and $\mathcal{M}_{2}^{2}$ summed over the signal region and over all bins. The values are fit to a constant. upper left: for the $\mathcal{M}_{s}^{2}$ summed over the signal region; upper right: for the $\mathcal{M}_{2}^{2}$ summed over the signal region; lower left: for the $\mathcal{M}_{s}^{2}$ summed over all bins; lower right: for the $\mathcal{M}_{2}^{2}$ summed over all bins. 